\title{
Relationship between diet and seborrheic dermatitis
}

\section{Funda Tamer}

\author{
Ufuk University School of Medicine, Department of Dermatology, Ankara, Turkey
}

Corresponding author: Dr. Funda Tamer, E-mail: fundatmr@yahoo.com

\begin{abstract}
Background: Seborrheic dermatitis is a chronic inflammatory skin disease that affects 1-10\% of the general population. Malassezia yeasts, immune response and emotional stress have been implicated in the etiology of seborrheic dermatitis. However, the effect of diet on development of seborrheic dermatitis is still controversial. This study investigates the differences in the frequency of consumption of foods and food groups between patients with seborrheic dermatitis and healthy individuals. Material and Methods: Fifty-one patients with seborrheic dermatitis of the scalp (28 female, 23 male) and 50 healthy individuals within the control group ( 30 female, 20 male) have been included in this study. The mean age of the patients and control group were $30.6 \pm 11.4$ years and $32.7 \pm 10.7$ years, respectively $(p=0.35)$. Dietary habits were evaluated by a questionnaire which includes frequency of intake of meat, processed meat, chicken, fish, egg, legume, milk, dairy products, fruit, vegetable, bread, tea, coffee, coke, fast food, sugar, pasta, rice, chocolate, cake, cookie and pie. The questionnaire included options like every day, 3-5 times a week, 1-2 times a week, twice a month, once a month and none. Results: The frequency of intake of foods in the questionnaire was simlar between patients and healthy individuals except for vegetables. Vegetable consumption was significantly more common among patients than healthy individuals $(\mathrm{p}=0.04)$. Conclusions: The results showed that dietary habits were not associated with increased risk of seborrheic dermatitis.
\end{abstract}

Key words: Dandruff; Diet; Seborrhea; Seborrheic dermatitis

\section{INTRODUCTION}

Seborrheic dermatitis is a chronic inflammatory skin disease which is characterised by erythematous scaly plaques on the sebaceous areas like forehead, eyebrows, glabella, nasolabial folds, scalp, chest, and back. The prevalence of seborrheic dermatitis among adults in the general population is $1-10 \%$. It is more common in men than in women. The disease usually presents between the ages of 30 and 40 years [1]. The incidence of seborrheic dermatitis is similar between ethnic groups [2]. The exact cause of this condition remains unknown. However, numerous factros including Malassezia yeasts, sex hormones like androgens, sebum levels, immune response, neurogenic factors and stress have been implicated in the etiopathogenesis of seborrheic dermatitis [3].

Sebaceous glands are distributed all over the skin surface except palms and soles. However, the secretion of sebum from sebaceous glands is highest on the scalp, face, chest, and back [4]. Sebum has an important role in the pathogenesis of seborrheic dermatitis [5]. It maintainces epidermal barrier function, carries anti-oxidants to the skin surface and protects skin from microbial colonization. Sebum is composed of triglycerides, fatty acids, wax esters, sterol esters, cholesterol, cholesterol esters, and squalene. Lipids are used by Malassezia species for proliferation. They degrade sebum, free fatty acids and leave the unsaturates which cause inflammation, irritation and scaling in seborrheic dermatitis [4].

Insulin and insulin-like growth factor-1 (IGF-1) stimulate the lipogenesis of sebaceous glands by a transcription factor named sterol regulatory elementbinding protein 1 . It has been suggested that high carbohydrate intake leads to reactive hyperinsulinemia and increased IGF-1 production [6]. Zinc, riboflavin, pyridoxine and niacin deficiency can lead to seborrheic dermatitis-like lesions. Therefore, nutrition has been associated with an increased risk of seborrheic 
dermatitis [2]. However, no exact relation has been established yet [7].

The purpose of this study was to examine the frequency of consumption of foods in patients with seborrheic dermatitis and healthy subjects and determine the relationship between dietary habits and seborrheic dermatitis.

\section{MATERIALS AND METHODS}

This study included 51 patients with seborrheic dermatitis of the scalp and 50 healthy individuals within the control group. The exclusion criteria were having an inflammatory skin disease like psoriasis, lichen planus, having a chronic condition like diabetes, hypertension, renal insufficiency, hypothyroidism, hyperthyroidism, obesity, cancer, eating disorder, and requiring a special diet.

Dietary intake was evaluated by a food frequency questionnaire which consisted of frequency of intake of meat, processed meat, chicken, fish, egg, legume, milk, dairy products, fruit, vegetable, bread, tea, coffee, coke, fast food, sugar, pasta, rice, chocolate, cake, cookie and pie. The questionnaire included options such as every day, 3-5 times a week, 1-2 times a week, twice a month, once a month and none. All participants answered the questionnaire based on their eating habit for the last five years.

Statistical analysis was performed using SPSS 22.0 (SPSS Inc., Chicago, IL). Continuous variables were defined as the means $( \pm)$ standard deviations and categorical variables as percentages. Differences between groups were analysed by independent-samples t-test for numerical variables and chi-square test for categorical variables. A p-value $<0.05$ was considered statistically significant.

\section{RESULTS}

Fifty-one patients with seborrheic dermatitis (28 female, 23 male) and 50 healthy individuals within

Table 1: Information about the frequency of consumption of some foods among patients and control group

\begin{tabular}{|c|c|c|c|c|c|c|}
\hline & & Patient $(n=51)$ & Groups & Control $(n=50)$ & & P-value \\
\hline \multirow[t]{6}{*}{ Meat } & None & 1 & $2 \%$ & 0 & $0 \%$ & 0.43 \\
\hline & Once a month & 4 & $7.8 \%$ & 4 & $8 \%$ & \\
\hline & Twice a month & 4 & $7.8 \%$ & 4 & $8 \%$ & \\
\hline & 1-2 times a week & 25 & $49 \%$ & 16 & $32 \%$ & \\
\hline & 3-5 times a week & 14 & $27.5 \%$ & 21 & $42 \%$ & \\
\hline & Everyday & 3 & $5.9 \%$ & 5 & $10 \%$ & \\
\hline \multirow[t]{6}{*}{ Milk } & None & 8 & $15.7 \%$ & 5 & $10 \%$ & 0.62 \\
\hline & Once a month & 6 & $11.8 \%$ & 7 & $14 \%$ & \\
\hline & Twice a month & 8 & $15.7 \%$ & 9 & $18 \%$ & \\
\hline & 1-2 times a week & 13 & $25.5 \%$ & 14 & $28 \%$ & \\
\hline & 3-5 times a week & 10 & $19.6 \%$ & 5 & $10 \%$ & \\
\hline & Everyday & 6 & $11.8 \%$ & 10 & $20 \%$ & \\
\hline \multirow[t]{6}{*}{ Fruit } & None & 0 & $0 \%$ & 1 & $2 \%$ & 0.68 \\
\hline & Once a month & 2 & $3.9 \%$ & 1 & $2 \%$ & \\
\hline & Twice a month & 3 & $5.9 \%$ & 1 & $2 \%$ & \\
\hline & 1-2 times a week & 3 & $5.9 \%$ & 5 & $10 \%$ & \\
\hline & 3-5 times a week & 17 & $33.3 \%$ & 19 & $38 \%$ & \\
\hline & Everyday & 26 & $51 \%$ & 23 & $46 \%$ & \\
\hline \multirow[t]{6}{*}{ Bread } & None & 2 & $3.9 \%$ & 1 & $2 \%$ & 0.93 \\
\hline & Once a month & 0 & $0 \%$ & 0 & $0 \%$ & \\
\hline & Twice a month & 1 & $2 \%$ & 1 & $2 \%$ & \\
\hline & 1-2 times a week & 5 & $9.8 \%$ & 4 & $8 \%$ & \\
\hline & 3-5 times a week & 8 & $15.7 \%$ & 6 & $12 \%$ & \\
\hline & Everyday & 35 & $68.6 \%$ & 38 & $76 \%$ & \\
\hline \multirow[t]{6}{*}{ Sugar } & None & 7 & $13.7 \%$ & 10 & $20 \%$ & 0.16 \\
\hline & Once a month & 2 & $3.9 \%$ & 4 & $8 \%$ & \\
\hline & Twice a month & 5 & $9.8 \%$ & 4 & $8 \%$ & \\
\hline & 1-2 times a week & 7 & $13.7 \%$ & 5 & $10 \%$ & \\
\hline & 3-5 times a week & 15 & $29.4 \%$ & 5 & $10 \%$ & \\
\hline & Everyday & 15 & $29.4 \%$ & 22 & $44 \%$ & \\
\hline \multirow[t]{6}{*}{ Vegetable } & None & 0 & $0 \%$ & 0 & $0 \%$ & 0.04 \\
\hline & Once a month & 0 & $0 \%$ & 2 & $4 \%$ & \\
\hline & Twice a month & 2 & $3.9 \%$ & 2 & $4 \%$ & \\
\hline & 1-2 times a week & 14 & $27.5 \%$ & 3 & $6 \%$ & \\
\hline & 3-5 times a week & 21 & $41.2 \%$ & 26 & $52 \%$ & \\
\hline & Everyday & 14 & $27.5 \%$ & 17 & $34 \%$ & \\
\hline
\end{tabular}


the control group (30 female, 20 male) were included in the study. The mean age of the patients and control group were $30.6 \pm 11.4$ years and $32.7 \pm 10.7$ years, respectively $(\mathrm{p}=0.35)$. The mean disease duration was $7.3 \pm 8.5$ years. Dietary habits were similar between the two groups except for vegetable intake (Table 1). Consumption frequency of vegetable was everyday in $14(27.5 \%)$ patients; $3-5$ times a week in $21(41.2 \%)$ patients; $1-2$ times a week in $14(27.5 \%)$ patients; twice a month in $2(3.9 \%)$ patients. However, vegetable consumption frequency was everyday in 17 (34\%) controls; 3-5 times a week in 26 (52\%) controls; 1-2 times a week in $3(6 \%)$ controls; twice a month in $2(4 \%)$ controls and once a month in $2(4 \%)$ controls. Vegetable consumption was significantly more frequent among patients than controls $(\mathrm{p}=0.04)$. There were no statistically significant differences between patients and healthy individuals in the frequency of intake of meat $(p=0.43)$, processed meat $(p=0.94)$, chicken $(p=0.33)$, fish $(\mathrm{p}=0.70)$, egg $(\mathrm{p}=0.14)$, legume $(\mathrm{p}=0.53)$, milk $(\mathrm{p}=0.62)$, dairy products; yogurt $(\mathrm{p}=0.28)$, cheese $(p=0.88)$, fruit $(p=0.68)$, bread $(p=0.93)$, tea, coffee $(p=0.20)$, coke $(p=0.13)$, fast food $(p=0.79)$, sugar $(\mathrm{p}=0.16)$, pasta, rice $(\mathrm{p}=0.66)$, chocolate $(\mathrm{p}=0.34)$, cake, cookie and pie $(\mathrm{p}=0.98)$.

\section{DISCUSSION}

Seborrheic dermatitis is characterized byhyperkeratosis, parakeratosis, excess intercellular and intracellular lipids. Epidermal keratinocytes consist of lipid granules which play role in the skin permeability. However, sebaceous glands are the main source of lipids of skin surface [6]. Therefore, sebaceous secretions are considered to play a pathogenetic role in seborrheic dermatitis [8]. Sebaceous glands provide lipid substrates for the Malassezia growth. Sebum consists of $57.5 \%$ triglycerides, $26.0 \%$ wax esters, $12.0 \%$ squalene, $3.0 \%$ cholesterol esters and $1.5 \%$ cholesterol [6]. Sebaceous gland secretions metabolise to irritating unsaturated fatty acids by Malassezia [4]. The prevelance of the disease is directly correlated with increased sebaceous gland activity [8]. Predilection sites are sebaceous gland rich areas like face, ears, scalp and the upper trunk [6]. Patients with oily skin are prone to develop seborrheic dermatitis [9]. When hygiene is poor as occurs in neuropathic patients, the residual sebum on the skin results in growth of Malassezia and seborrheic dermatitis [10].
The effect of diet on increased sebum production has been reported previously. It has been suggested that dietary lipids like fatty acids, acetat and glucose may be the source for sebum synthesis [6]. Frequent consumption of carbohydrates with a high glycemic index can lead to hyperinsulinemia. It istimulates sebum secretion by increasing androgen levels [11]. Boelsma et al. investigated the effect of diet on the skin conditions including hydration, sebum content, and surface $\mathrm{pH}$ of the skin in 302 healthy subject. They assessed dietary intake with food frequency questionnaires and sebum content of the forehead with the sebumeter. No association between the nutrients in diet and sebum content of the skin have been found. However, increased serum vitamin A levels were related with decreased sebum content [12]. It has been suggested that caloric restriction can reduce sebum release. Pochi et al. evaluated sebaceous gland activity in obese patients following a four to eight week-long caloric deprivation. The study showed decrease in sebaceous gland secretion in all patients [13].

Dietary changes shown to be benefical in the treatment of skin diseases like psoriasis, scleroderma, acne, rosacea, herpes, pemphigus, and Refsum's disease [14]. Moreover, dietary recommendations have been reported in the treatment of dandruff which is regarded as a mild form of seborrheic dermatitis with scalp scaling $[15,16]$. Patients with dandruff were adviced to eat foods rich in vitamin $B$, shellfish, red meat, sunflower seeds, sardines, salmon, water based fruits and vegetables. In addition to this, the patients were adviced to avoid oily and greasy food products, sugar, junk foods, animal fats, flour, and seafood [16]. Faulty eating habits have also been reported to be related with seborrheic dermatitis [9]. In 1967, Bett et al. measured the sugar intake of 16 patients with seborrheic dermatitis and two groups of control subjects with a questionary. A control group included 16 healthy individuals and the other included 16 patients suffering from warts. The consumption of sugar by patients with seborrheic dermatitis was found to be significantly higher than two groups of control subjects [17].

However, the association between diet and seborrheic dermatitis has not been clearly established yet. There are no adequate number of studies in the literature. Therefore, frequency of food consumption of patients with seborrheic dermatitis and healthy individuals have been compared in this study. No significant differences have been observed between two groups except for frequency of vegetable intake. Vegetable consumption 
was significantly more frequent among patients than controls. This study indicates that diet doesn't play role in production of sebum in patients with seborrheic dermatitis.

\section{CONCLUSION}

In conclusion, the results showed that dietary habits were not associated with increased risk of seborrheic dermatitis.

\section{STATEMENT OF HUMAN AND ANIMAL RIGHTS}

All procedures followed were in accordance with the ethical standards of the responsible committee on human experimentation (institutional and national) and with the Helsinki Declaration of 1975, as revised in 2008.

\section{STATEMENT OF INFORMED CONSENT}

Informed consent was obtained from all patients for being included in the study.

\section{REFERENCES}

1. Ijaz N, Fitzgerald D. Seborrhoeic dermatitis. Br J Hosp Med. 2017;78:88-91.

2. Borda LJ, Wikramanayake TC. Seborrheic dermatitis and dandruff: a comprehensive review. J Clin Investig Dermatol. 2015;3:10.13188/2373-1044.1000019.

3. Bukvić Mokos Z, Kralj M, Basta-Juzbašić A, Lakoš Jukić I. Seborrheic dermatitis: an update. Acta Dermatovenerol Croat. 2012;20:98-104.
4. Ro BI, Dawson TL. The role of sebaceous gland activity and scalp microfloral metabolism in the etiology of seborrheic dermatitis and dandruff. J Investig Dermatol Symp Proc. 2005;10:194-7.

5. Inaloz HS, Kirtak N. The pathogenesis and treatment of seborrhoeic dermatitis. T Klin J Med Sci. 2002;22:239-44.

6. Sakuma TH, Maibach HI. Oily skin: an overview. Skin Pharmacol Physiol. 2012;25:227-35.

7. Schwartz RA, Janusz CA, Janniger CK. Seborrheic dermatitis: an overview. Am Fam Physician. 2006;74:125-30.

8. Bakardzhiev Argirov A, Bakardzhiev I. New insights into the etiopathogenesis of seborrheic dermatitis. Clin Res Dermatol Open Access. 2017;4:1-5.

9. MacKee GM, Lewis GM. Dandruff and seborrhea: I. flora of "normal" and diseased scalps. J Invest Dermatol. 1938;1:131-9.

10. Sampaio AL, Mameri AC, Vargas TJ, Ramos-e-Silva M, Nunes AP, Carneiro SC. Seborrheic dermatitis. An Bras Dermatol. 2011;86:1061-71.

11. Costa A, Lage D, Moisés TA. Acne and diet: truth or myth? An Bras Dermatol. 2010;85:346-53.

12. Boelsma E, van de Vijver LP, Goldbohm RA, Klöpping-Ketelaars IA, Hendriks HF, Roza L. Human skin condition and its associations with nutrient concentrations in serum and diet. Am J Clin Nutr. 2003;77:348-55.

13. Pochi PE, Downing DT, Strauss JS. Sebaceous gland response in man to prolonged total caloric deprivation. J Invest Dermatol. 1970;55:303-9.

14. Basavaraj KH, Seemanthini C, Rashmi R. Diet in dermatology: present perspectives. Indian J Dermatol. 2010;55:205-10.

15. Dessinioti C, Katsambas A. Seborrheic dermatitis: etiology, risk factors, and treatments: facts and controversies. Clin Dermatol. 2013;31:343-51.

16. Mohamed HS, Farahat NH, Megallaa NG, Elhaleem MA. Nursing guidelines on hair dandruff symptoms for adult patients. Life Sci J. 2014;11:323-33.

17. Bett DG, Morland J, Yudkin J. Sugar consumption in acne vulgaris and seborrhoeic dermatitis. Br Med J. 1967;3:153-5.

Copyright by Funda Tamer. This is an open-access article distributed under the terms of the Creative Commons Attribution License, which permits unrestricted use, distribution, and reproduction in any medium, provided the original author and source are credited.

Source of Support: Nil, Conflict of Interest: None declared. 